\title{
Effect of surface roughness on the angular acceleration for a droplet on a super-hydrophobic surface
}

\author{
Longyang LI ${ }^{1}$, Jingfang ZHU ${ }^{2}$, Zhixiang ZENG ${ }^{1, *}$, Eryong LIU ${ }^{3}$, Qunji XUE ${ }^{1}$ \\ ${ }^{1}$ Key Laboratory of Marine Materials and Related Technologies, Zhejiang Key Laboratory of Marine Materials and Protective \\ Technologies, Ningbo Institute of Materials Technology and Engineering, Chinese Academy of Sciences, Ningbo 315201, China \\ ${ }^{2}$ SEU-FEI Nano-Pico Center, Key Laboratory of MEMS of Ministry of Education, Southeast University, Nanjing 210096, China \\ ${ }^{3}$ School of Materials Science and Engineering, Xi'an University of Science and Technology, Xi'an 710054, China \\ Received: 01 November 2019 / Revised: 04 March 2020 / Accepted: 27 March 2020 \\ (C) The author(s) 2020.
}

\begin{abstract}
The motion of droplets on a super-hydrophobic surface, whether by sliding or rolling, is a hot research topic. It affects the performance of super-hydrophobic materials in many industrial applications. In this study, a super-hydrophobic surface with a varied roughness is prepared by chemical-etching. The adhesive force of the advancing and receding contact angles for a droplet on a super-hydrophobic surface is characterized. The adhesive force increases with a decreased contact angle, and the minimum value is $0.0169 \mathrm{mN}$ when the contact angle is $151.47^{\circ}$. At the same time, the motion of a droplet on the superhydrophobic surface is investigated by using a high-speed camera and fluid software. The results show that the droplet rolls instead of sliding and the angular acceleration increases with an increased contact angle. The maximum value of the angular acceleration is $1,203.19 \mathrm{rad} / \mathrm{s}^{2}$ and this occurs when the contact angle is $151.47^{\circ}$. The relationship between the etching time, roughness, angular acceleration, and the adhesion force of the forward and backward contact angle are discussed.
\end{abstract}

Keywords: super-hydrophobic surface; adhesive force; rolling friction; contact angle

\section{Introduction}

In the industrial field, there are several great potential applications of super-hydrophobic surfaces. Some examples include drag reduction [1, 2], lubrication [3], anticorrosion [4-6], self-cleaning [7, 8], and antiicing [9]. As a result, the design, preparation, application, and theoretical model of super-hydrophobic systems have investigated $[8,10,11]$. There are many techniques that can fabricate super-hydrophobic surfaces, which include sol-gel [12-14], chemical-etching [15-17], chemical vapor deposition (CVD) [18], anodicoxidation [19, 20], and vapor-deposition [21, 22]. Super-hydrophobic surfaces are commonly defined by having a contact angle greater than $150^{\circ}$ and the rolling angle is less than $10^{\circ}$ when the droplet contacts the solid surface. Although many factors influence super-hydrophobicity, surface roughness and surface energy are the dominant factors. To improve the hydrophobicity, it is necessary to provide an appropriate surface roughness for the materials with a low surface energy.

There are many movement models of droplets on a super-hydrophobic surface. These include bouncing [23, 24], rolling [25-29], and sliding [30-32], however, they are controversial [11, 33-35]. Many researchers consider that the motion of the droplet on a super-hydrophobic material involves sliding and the sliding angle is only related to the contact angle. The sliding angle increases with a decreasing

* Corresponding author: Zhixiang ZENG, E-mail: zengzhx@nimte.ac.cn 
contact angle [10, 31, 33, 36]. For instance, Liang et al. [31] fabricated a super-hydrophobic surface on high-speed steel by chemically modifying it. For this super-hydrophobic surface, they found that the contact angle was $157^{\circ}$ and the sliding angle was $2.8^{\circ}$. Miwa et al. [37] coated heptadecafluorodecyltrimethoxysilane onto glass and fabricated a superhydrophobic surface with a contact angle of $160^{\circ}$ and a sliding angle of $1^{\circ}$ for a $7 \mathrm{mg}$ water droplet. Feng et al. [38] prepared a super-hydrophobic surface on a carbon nanotube with a contact angle of $166^{\circ}$ and a sliding angle of $3^{\circ}$ for a water droplet. They believed that an increased surface roughness and an enlarged air-liquid interface fraction led to a reduction in the wettability and decreased the adhesivity on the surface. However, other researchers have obtained different results. For example, Ning et al. [32] demonstrated that a dodecafluoroheptyl methacrylate surface sprayed with silanized nanosilica had a contact angle of $152.2^{\circ}$, however, the droplet adhered to the surface. They suggested that an ultrahigh adhesive force is due to the chemical composition, especially for hydroxyl groups and the surface roughness.

On the other hand, other researchers believe that droplets roll on super-hydrophobic surfaces. Yao et al. [29] fabricated a super-hydrophobic surface on silicon wafers. They investigated different droplet sizes and the contact angle hysteresis impact on the rolling angle. Their results revealed that the rolling angle decreased along with an increase in the droplet size and the spacing between the micropillars. Marmur et al. [27] investigated the relationship between the main parameters for super-hydrophobicity. They determined that the roll angle decreased along with the increase of contact angle. In addition, they reported that the droplet cannot roll on the super-hydrophobic surface when in the Wenzel state. To date, there are a few studies that focus on the relationship between the rolling angle, the contact angle, and the adhesive force that correspond to the advancing contact angle (ACA) and receding contact angle (RCA). As a result, the droplet rolling properties for super-hydrophobic surfaces is not completely understood.

Currently, super-hydrophobic surfaces with variations in the surface roughness can be fabricated through chemical-etching. In addition, the relationships between the rolling angle, contact angle, and the surface structure are presently explored. The adhesive force of the ACA and RCA is characterized by a dynamic contact-angle measuring device and a tensiometer with a high-speed camera. The form of motion for a droplet on a super-hydrophobic surface is observed with a high-speed camera. At the same time, the droplet's motion is studied with visualization technology by applying fluid software simulations. Based on a new theoretical model, the influence of the etching time on angular acceleration is discussed.

\section{Experimental}

\subsection{Surface fabrication and characterization}

The performance of a super-hydrophobic surface is not only dependent on the surface energy, but also on the roughness of the surface. Therefore, samples with a varied surface roughness are fabricated by etching with hydrochloric acid on aluminum sheets $(99.3 \%, 120 \mathrm{~mm} \times 50 \mathrm{~mm} \times 4 \mathrm{~mm})$. This is achieved by using deionized water (DI) and hydrochloric acid $(\mathrm{HCl} 30 \%)$ with a volume ratio of $4: 1$, and the surface roughness is controlled by the etching time. In order to obtain a clean surface, the aluminum sheets are cleaned several times in DI by using an ultrasonic method. Then, the aluminum sheets are placed in an oven at $100{ }^{\circ} \mathrm{C}$ for approximately $20 \mathrm{~min}$. The clean, dry aluminum sheets are then immersed in a lauric acid ethanol solution $\left(\mathrm{CH}_{3}\left(\mathrm{CH}_{2}\right)_{10} \mathrm{COOH}\right.$, $0.4 \mathrm{~mol} / \mathrm{L}$ ) at $60{ }^{\circ} \mathrm{C}$. The sample is then removed after $6 \mathrm{~h}$. Finally, super-hydrophobic surfaces are obtained by performing rinsing with DI and drying in a vacuum oven at $90{ }^{\circ} \mathrm{C}$ for $2 \mathrm{~h}$. A field emission scanning electron microscope (FE-SEM, FEI Quanta 250 FEG, USA) and laser-scanning confocal microscopy (Zeiss LSM700, Germany) are used to characterize the surface topography and the surface roughness of the samples. The chemical composition of the samples is characterized by using X-ray photoelectron spectroscopy (XPS Axis Ultra DLD, Kratos Co., Ltd., UK). The influence of wettability and adhesion of the obtained samples for the surface roughness is investigated by using a video-based optical system (Dataphysics OCA25, Germany) and a dynamic contact-angle measuring device and tensiometer 
(Dataphysics DCAT21, Germany) with a high-speed camera (photron fastcam MiNi 100, Photron Co., Ltd., Japan). These technologies can measure the ACA, RCA, and the apparent contact angle for at least five different positions in every sample. First, a droplet with a volume of $3 \mu \mathrm{L}$ is placed in a metal circle with a diameter of approximately $2 \mathrm{~mm}$. Subsequently, the obtained samples are placed on a stage that can move up and down. The stage moves up until the droplet $(3 \mu \mathrm{L})$ achieves contact with the obtained sample surfaces. The stage then moves down until the droplet $(3 \mu \mathrm{L})$ is completely separated from the sample surfaces. At the same time, the high-speed camera records the entire process of the adhesion test for the sample surfaces. This process can determine the adhesion force that corresponds to the ACA and RCA, as shown in Fig. 1.

\subsection{Simulation setting}

To explore whether the droplets are rolling or sliding on the super-hydrophobic surfaces, the prepared sample is tilted $15^{\circ}$ and the droplet with a radius of $2 \mathrm{~mm}$ is placed on top of the sample. At the same time, the entire motion state of the droplet is recorded with a high-speed camera. The high-speed camera is operated at 2,000 frames per second. In the numerical simulation, the computational domain is simplified in Fig. 2. The length, height, and width of the computational domain are 30,6 , and $3 \mathrm{~mm}$, respectively. The fluid medium is set as water with a density of $998.2 \mathrm{~kg} / \mathrm{m}^{3}$ and a viscosity of $0.001003 \mathrm{~Pa} \cdot \mathrm{s}$. The grid spacing increases from the walls by an aspect ratio of 1.1, which results in a structured mesh; the first distance from the wall is $1 \times 10^{-7} \mathrm{~m}$. The velocity of the inlet is set to zero and the outlet is set as the pressure outlet. In order to verify the grid independence, the grid number is set as 1E6, 6E6, and 9E6. In comparison to 1E6, the droplet's displacement increment is $4.35 \%$ for $6 \mathrm{E} 6$ and $4.36 \%$ for 9E6, respectively. To save resources and the computation time, the 6E6 grid is chosen by the computer as shown in Fig. 2. The volume of fluid (VOF) model combined with the continuous surface force (CSF) is selected. In the VOF model, the surface tension coefficient is set as $0.072 \mathrm{~N} / \mathrm{m}$ and the contact angle is set as $169^{\circ}$. In addition, the droplet does not spread on the surface of the sample and the length of the contact line is constant. As a result, the dynamic contact angle has little effect on the movement of the droplets. To achieve a fast convergence in the VOF model by combining an implicit solution with an algebraic multigrid method. This high accuracy result is obtained by using a second-order upwind scheme to discretize all of the equations. Continuous monitoring is performed for the residual continuity, $x_{-}, y^{-}$, and $z$-velocity. All of the residual values are less than $1 \times 10^{-6}$ and the solution is assumed to converge. The other wall surface is set to be symmetric to reduce the effect of the wall surface on the droplets. To ensure convergence for the computation, a time
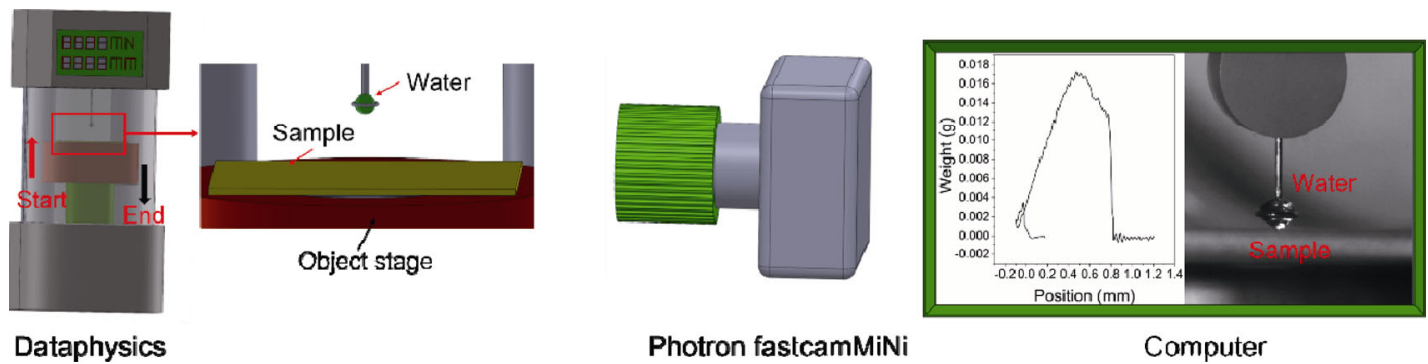

Fig. 1 Schematics of the process used to measure the adhesive force.

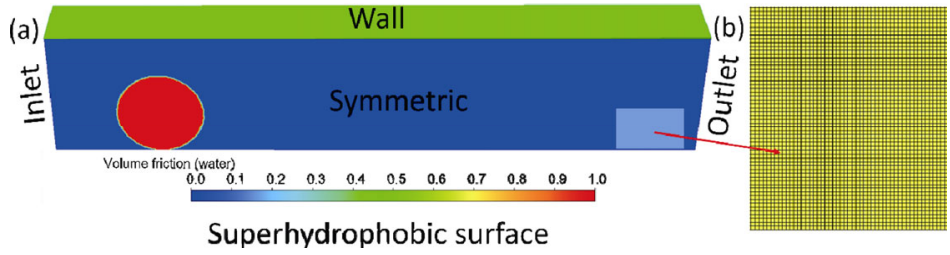

Fig. 2 (a) Computational initialization and computational domain. (b) Enlargement of the grids for the cross-section. 
step size $(\Delta t)$ of $1 \times 10^{-6} \mathrm{~s}$ is used, and the other parameters are set to the default values.

\section{Results and discussion}

Figure 3 shows the surface topographies of the samples, which have different etching time and are decorated with lauric acid. A great number of micro- and/or nano-scale structures appear on the surface of the samples. At the same time, with an increase in the etching time, the number of micro- and/or nano-scale structures increased. The surface roughness of the sample varies with the etching time. The surface with a lauric acid coating has a gradually increasing surface roughness from 9.734 to $12.307 \mu \mathrm{m}$ along with an etching time from 1 to $15 \mathrm{~min}$. The surface roughness drastically increases to $20.406 \mu \mathrm{m}$ as the etching time further increases to $25 \mathrm{~min}$ (Fig. 4).
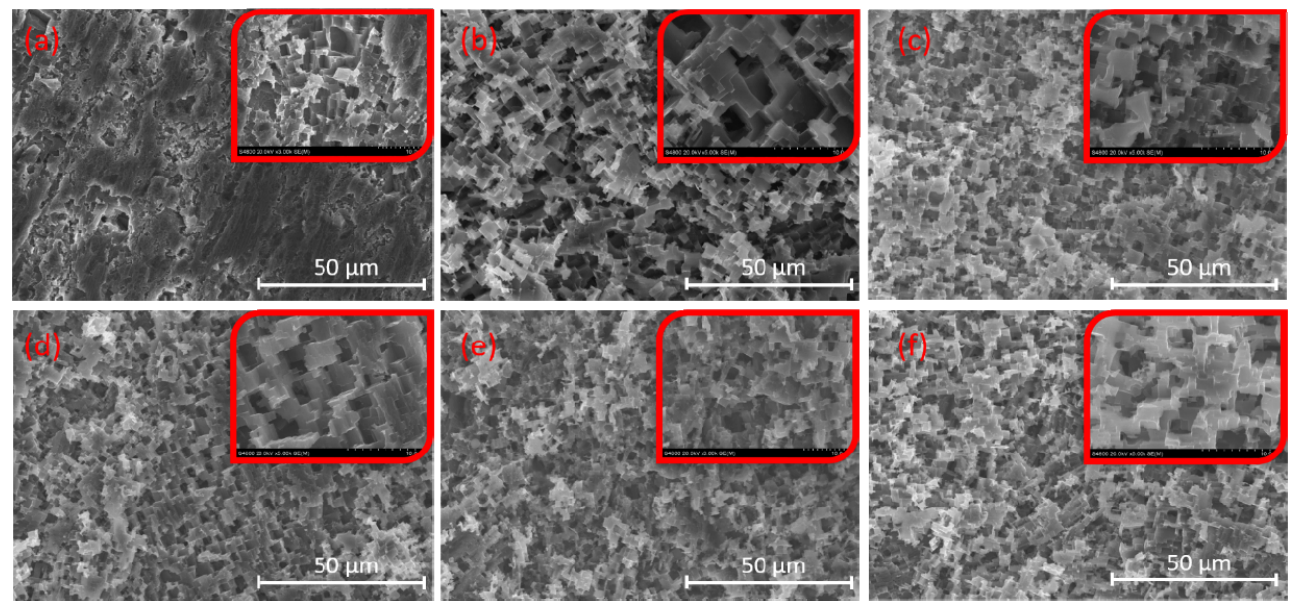

Fig. 3 SEM images of the surface morphology with hierarchical structures fabricated using different etching time of (a) 1 min, (b) $5 \mathrm{~min}$, (c) $10 \mathrm{~min}$, (d) $15 \mathrm{~min}$, (e) $20 \mathrm{~min}$, and (f) $25 \mathrm{~min}$, respectively.
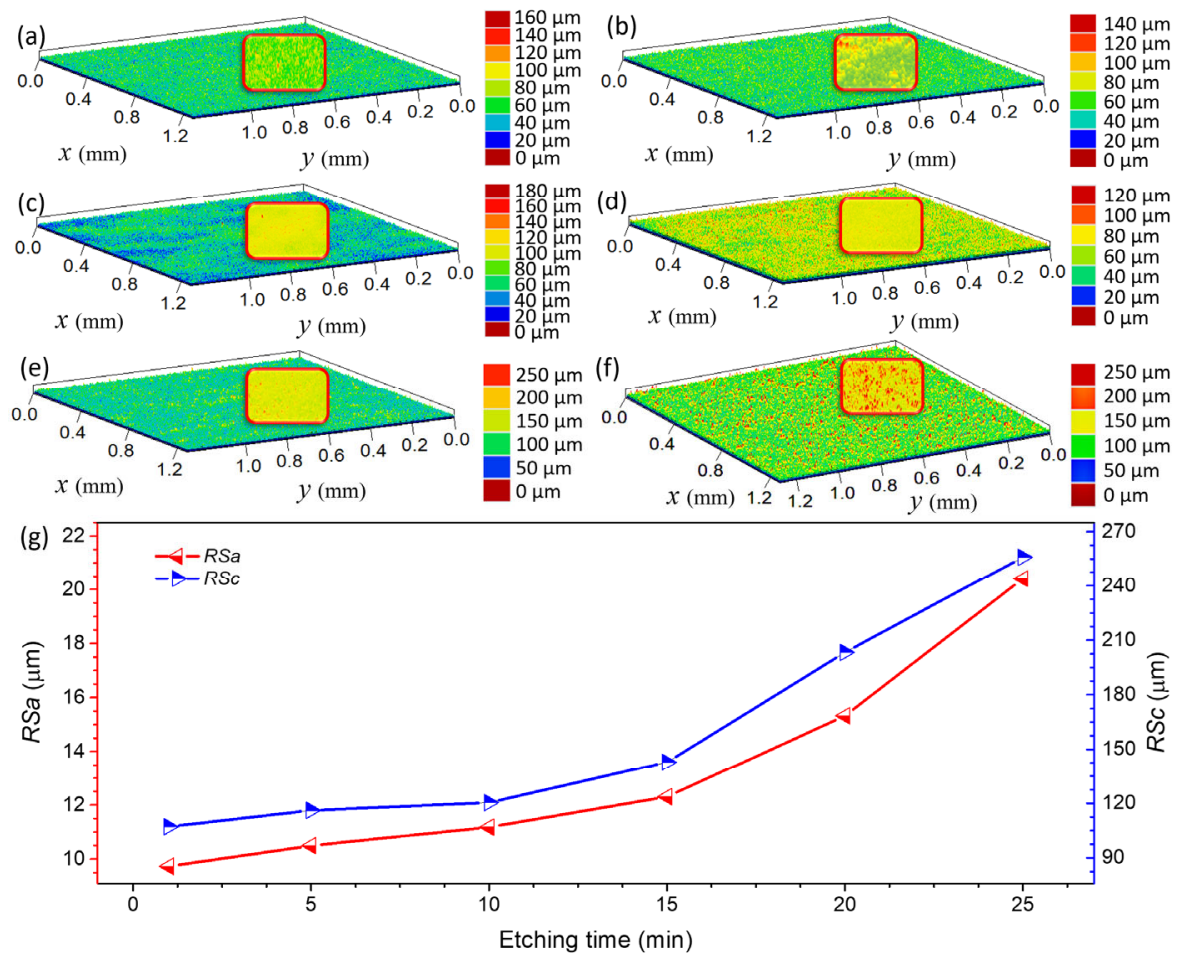

Fig. 4 Three-dimensional (3D) topographic profiles for different etching time: (a) $1 \mathrm{~min}$, (b) $5 \mathrm{~min}$, (c) $10 \mathrm{~min}$, (d) $15 \mathrm{~min}$, (e) $20 \mathrm{~min}$, and (f) $25 \mathrm{~min}$, and (g) the surface roughness of the sample as a function of the etching time. $R S a$ and $R S c$ represent the average surface roughness and mean of the height, respectively. 
The average deviation $R S a$ height of the randomlytextured surfaces for all of the surfaces is defined by Eqs. (1) and (2):

$$
\begin{aligned}
& R S a=\frac{1}{N_{x} N_{y}} \sum_{i=1}^{N_{x}} \sum_{j=1}^{N_{y}}\left[Z\left(x_{i}, y_{j}\right)-R S c\right] \\
& R S c=\frac{1}{N_{x} N_{y}} \sum_{i=1}^{N_{x}} \sum_{j=1}^{N_{y}} Z\left(x_{i}, y_{j}\right)
\end{aligned}
$$

where $N_{x}$ and $N_{y}$ represent the number of pixels in the $x$ - and $y$-directions, respectively. $x_{i}=0-1.5 \mathrm{~mm}$, $y_{i}=0-1.5 \mathrm{~mm}$, and $Z$ is the vertical distance from the substrate of surface. The results are shown in Figs. 3(a)-3(f). The main reason for the fast increase in the roughness is that a large amount of heat is released from the reaction with an increased etching time. This promotes an increase in the reaction rate; therefore, the surface roughness drastically increases as the etching time increases from 15 to $25 \mathrm{~min}$.

Two examples of wetting models for a droplet in contact with rough surfaces include the Wenzel model and the Cassie-Baxter model [39, 40].

$$
\begin{aligned}
& \cos \theta=r \cos \theta_{\mathrm{e}} \\
& \cos \theta=\lambda_{\mathrm{s}}\left(1+\cos \theta_{\mathrm{e}}\right)-1 \\
& \lambda_{\mathrm{s}}+\lambda_{\mathrm{g}}=1
\end{aligned}
$$

where $\theta$ represents the apparent contact angle; $\theta_{\mathrm{e}}$ represents the contact angle on the smooth surface; $\lambda_{\mathrm{s}}$ represents the area fraction of the liquid-solid interface; $\lambda_{\mathrm{g}}$ represents the area fraction of the liquid-gas interface; and $r$ represents the non- dimensional surface roughness, which is the ratio of the real surface area to the projected surface area. According to Eq. (3), when the surface roughness increases, hydrophobic surfaces tend to become more hydrophobic $\left(\theta_{e}>90^{\circ}\right)$. To investigate how the roughness affects the property of a super-hydrophobic surface, the samples are modified with lauric acid to decrease the surface energy to improve the contact angle on a smooth surface $\left(\theta_{e}=98^{\circ}\right)$; the results are presented in Fig. 5(a). According to Eqs. (3)-(5), when the surface roughness increases, the contact area of the droplet with the solid surface decreases. Hence, increasing the surface roughness will cause more air to remain on the surface. Under the action of low surface energy, it is difficult for the droplet to enter the structure and exclude air from the structure. Therefore, the liquid-solid contact transforms into a liquid-gas contact. The area of the liquid-gas and the contact angle of the droplet increases along with increasing the roughness. The maximum contact angle and the area fraction of the liquid-gas interface are $151.47^{\circ} \pm 1^{\circ}$ and $85.28 \%$, respectively, which occurs at $25 \mathrm{~min}$. These results are displayed in Fig. 5(b). The standard deviation $\left(s_{d}\right)$ of the sample for the apparent contact angle is calculated using Eqs. (6) and (7).

$$
\begin{aligned}
& \bar{x}=\frac{\sum_{i=1}^{N} x_{i}}{N} \\
& s_{d}=\sqrt{\frac{\sum_{i=1}^{N}\left(x_{i}-\bar{x}\right)^{2}}{N-1}}
\end{aligned}
$$
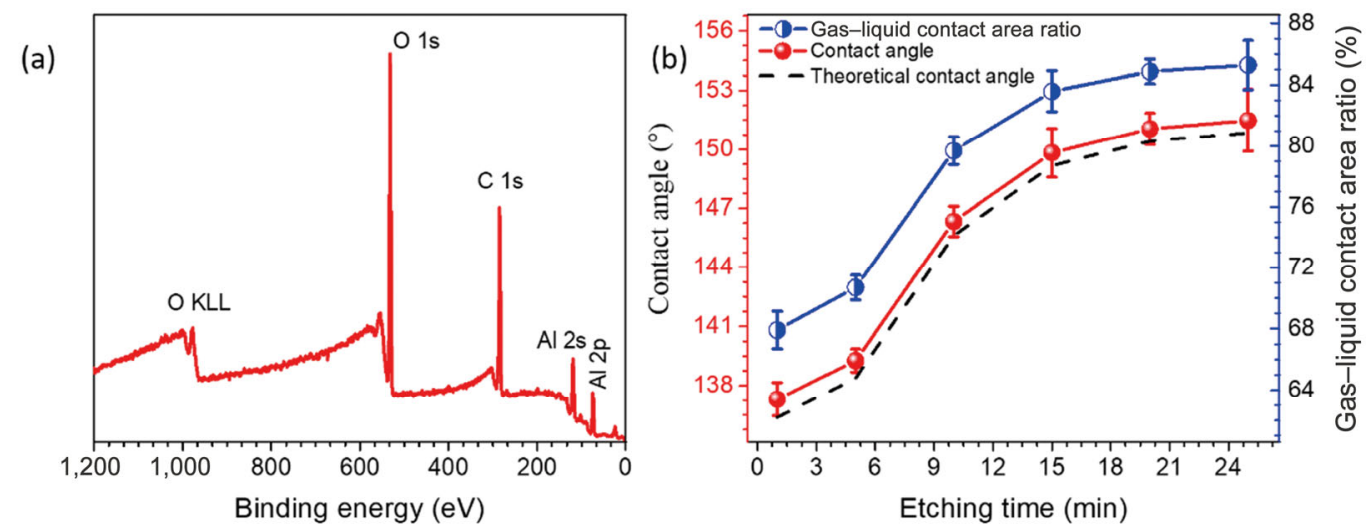

Fig. 5 (a) X-ray photoelectron spectroscopy of the as-prepared samples. (b) The theory [40] and measurement of the contact angle for a droplet plotted on the left $y$-axis and the gas-liquid contact-area ratio plotted on the right $y$-axis as a function of the etching time. 
where $\left(x_{1}, x_{2}, x_{3}, \cdots, x_{N}\right)$ represents the contact angle of the sample, $\bar{x}$ represents the mean value of these observations, and $N$ represents the number of observations of the sample. The deviation is mainly caused by an uneven roughness.

During the actual conditions, since the surface is not atomically smooth or chemically homogeneous, a droplet will have contact angle hysteresis when contacting the surface, which determines whether the droplet rolls easily or not on the surface. As demonstrated in Fig. 6, the contact angle hysteresis decreases along with an increase in the etching time. This is mainly because the contact radii of the droplets on the sample decreases with an increase in the apparent contact angle. The vertical distance from the center of the mass to the surface of the sample rises with an increasing apparent contact angle. Therefore, the sample is slightly inclined and the droplet will roll on the sample's surface under the action of gravity as depicted in Fig. 6. The ACA, $\mathrm{RCA}$, and the rolling angle are $155^{\circ}, 149^{\circ}$, and $5.8^{\circ}$ for an etching time of $25 \mathrm{~min}$, respectively. The ACA $\left(\theta_{\mathrm{A}}\right)$ can be calculated from the apparent contact angle $(\theta)$ and the RCA $\left(\theta_{\mathrm{R}}\right)$, which was put forward by Tadmor [41], and it was experimentally confirmed by Chibowski and Terpilowski [42] by using Eqs. (8)-(10).

$$
\begin{aligned}
& \theta=\arccos \left(\frac{r_{\mathrm{A}} \cos \theta_{\mathrm{A}}+r_{R} \cos \theta_{\mathrm{R}}}{r_{\mathrm{A}}+r_{\mathrm{R}}}\right) \\
& r_{\mathrm{A}}=\left(\frac{\sin ^{3} \theta_{A}}{2-3 \cos \theta_{\mathrm{A}}+\cos ^{3} \theta_{\mathrm{A}}}\right)^{1 / 3} \\
& r_{\mathrm{B}}=\left(\frac{\sin ^{3} \theta_{\mathrm{B}}}{2-3 \cos \theta_{\mathrm{B}}+\cos ^{3} \theta_{\mathrm{B}}}\right)^{1 / 3}
\end{aligned}
$$

In Fig. 1, the stage is slowly raised and then the object stage extrudes the droplet. The adhesive force and ACA will change along with the rising object stage and the adhesive force corresponding to the ACA is obtained by the high-speed camera. When the object stage arrives at the highest point, the object stage is slowly lowered. Then, the object stage draws the droplet and the adhesive force, and RCA will change as the object stage is lowered. As a result, the adhesive force corresponding to the RCA using the high-speed camera can be obtained. The absolute value of the adhesive force corresponding to the ACA decreases along with an increase in the etching time. The contact radii for the droplets on the sample also decrease with an increase in the etching time. The minimum values for the adhesive force and the radius are $0.0425 \mathrm{mN}$ and $0.217 \mathrm{~mm}$, respectively, as demonstrated in Fig. 7 . However, the adhesive force per unit of length increases with an increase in the etching time; the maximum value is $0.031 \mathrm{mN} / \mathrm{mm}$ as presented in Fig. 6(a). This increase occurs because the apparent contact angle increases along with the increase in the etching time for a low surface energy condition This can trap a substantial amount of air along the super-hydrophobic surface. The entrapped air prevents water from contacting the surface of the sample and this leads to a reduction in the wettability and a decreased adhesivity of the surface. Therefore, the roll angle decreases along with the increase of etching time, as presented in Fig. 6(b), however, the resistance of the water contact surface will increase. The variation in the adhesive force corresponding to the RCA and the contact radii for the droplets on the sample with the etching time are the same. This is because the superficial area becomes enlarged with an increased surface roughness, and the surface roughness increases along with an increase in the etching time. The actual contact area of the droplets on the sample surface increases along with an increase in the apparent contact angle when the water completely contacts the sample surface at the same contact radii. Therefore, the adhesive force per unit length corresponding to the RCA increases with an increase in the etching time. The minimum value of the adhesive force and the radii are $0.0169 \mathrm{mN}$ and $0.1199 \mathrm{~mm}$, respectively, as demonstrated in Fig. 6. The maximum value is $0.022 \mathrm{mN} / \mathrm{mm}$, as illustrated in Fig. 6(a).

As shown in Fig. 8, the radius of the sphere $(R)$ decreases with an increase in the contact angle, and the minimum value is $2.007 \mathrm{~mm}$ for an apparent contact angle of $151.47^{\circ} \pm 1^{\circ}$. The contact radius of the droplet on the surface of the sample $(r)$ decreases with an increased apparent contact angle; the minimum value is $0.958 \mathrm{~mm}$ for an apparent contact angle of 

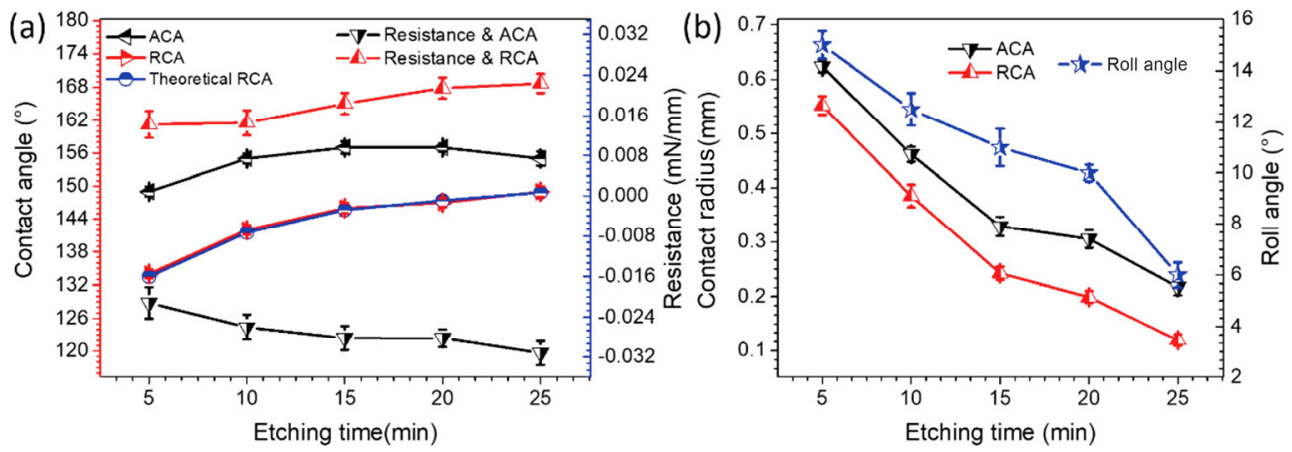

Fig. 6 (a) ACA, RCA, and the theoretical RCA [42] are plotted on the left $y$-axis, and the adhesive force per unit of length for the RCA and ACA is plotted on the right $y$-axis as a function of the etching time. (b) The contact radii for the droplets corresponding to the ACA and RCA are plotted on the left $y$-axis and the rolling angle is plotted on the right $y$-axis on the surface sample as a function of the etching time.
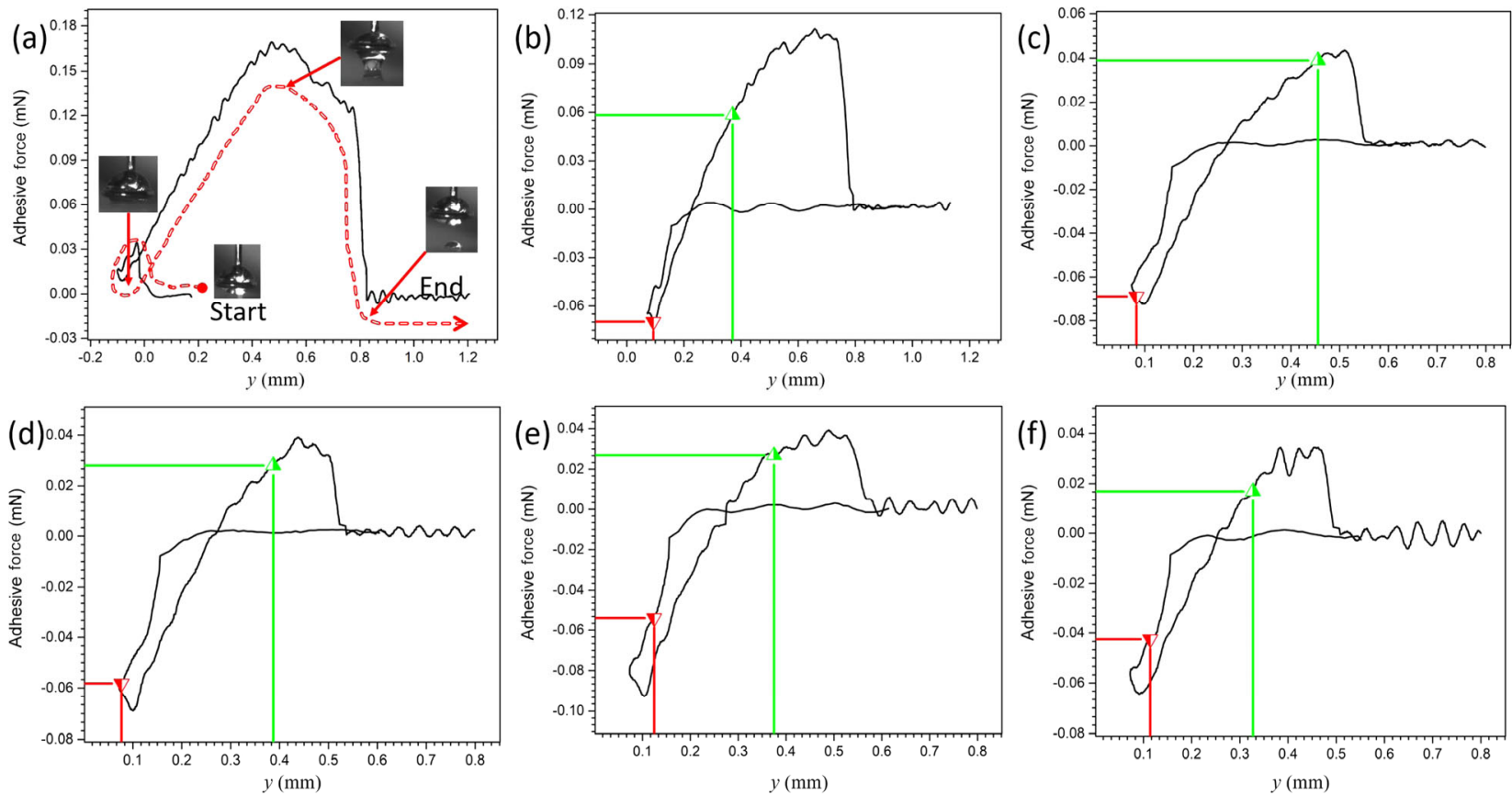

Fig. 7 (a) Schematic of the process used for measuring the apparent contact angle and the adhesive force corresponding to the RCA ( $\triangle$ ) and ACA ( $\nabla$ ) at different etching times: (b) $5 \mathrm{~min}$, (c) $10 \mathrm{~min}$, (d) $15 \mathrm{~min}$, (e) $20 \mathrm{~min}$, and (f) 25 min, respectively.

$151.47^{\circ} \pm 1^{\circ}$. The droplet on the surface can be regarded as a solid hemisphere with a very small bottom shape, which is small enough to ignore the deformation that is caused by gravity. In the ideal case, there are no hysteretic phenomena for the contact angle; the advancing angle and the receding angle have an equivalent value. The $R$ increases when the droplet contact surface of the sample increases. For droplets with a given mass $(m)$, the $r$ of the contact circle varies with an apparent contact angle. The relationship between $R$ and $r$ can easily be deduced by mathematical methods as shown in the following equations:

$$
\frac{4}{3} \pi R^{\prime 3} \rho g=m g
$$

$$
\begin{aligned}
R^{\prime} & =\left[\left(\frac{1}{4}\right)\left(2-3 \cos \theta+\cos ^{3} \theta\right)\right]^{1 / 3} \times R \\
r & =R \times \sin \theta
\end{aligned}
$$

For a given uniform sphere, the center of the sphere (point $H$ ) coincides with the center of the mass (point $H^{\prime}$ ) and the radius $\left(R^{\prime}\right)$ of the droplet (Fig. 8). However, if the bottom of the droplet is flattened, the center of the sphere and the center of mass is different. Figure 8 shows alternative cases for a droplet on a hydrophobic surface $\left(\theta \geq 90^{\circ}\right)$ where $h<0$. In Fig. 8, $\mathrm{OH}^{\prime}$ represents the height between the center of the mass and the surface of the solid, and $\theta$ represents the apparent contact angle. In addition, 

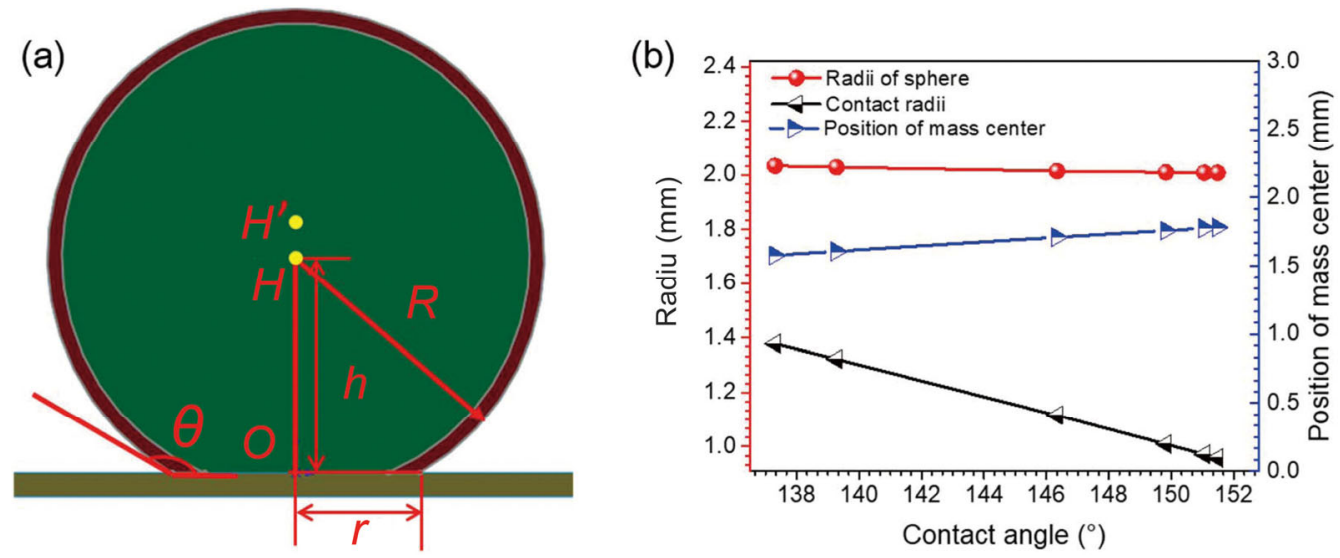

Fig. 8 (a) Centers of the sphere and mass. (b) The vertical position of the mass center from the sample's surface is plotted on the left $y$-axis. The contact radius with the sample's surface and the sphere of the droplet is plotted on the right $y$-axis as a function of the contact angle, respectively.

$h$ represents the distance from the center of a sphere to the surface of a solid surface. The distance between the two centers and the mass center can easily be deduced by a mathematical method as shown by the following equations:

$$
\begin{aligned}
\mathrm{d} V & =\pi \times\left(R^{2}-h^{2}\right) \mathrm{d} h \\
V & =\int_{h}^{R} \pi \times\left(R^{2}-h^{2}\right) \mathrm{d} h \\
H H^{\prime} & =\frac{\int_{h}^{R} h \times \pi \times\left(R^{2}-h^{2}\right) \mathrm{d} h}{\int_{h}^{R} \pi \times\left(R^{2}-h^{2}\right) \mathrm{d} h} \\
O H^{\prime} & =H H^{\prime}-h \\
h & =R \cos \theta \\
O H^{\prime} & =R \times \frac{4-(1+\cos \theta)^{2}}{4 \times(2+\cos \theta)}
\end{aligned}
$$

Therefore, the vertical distance from the mass center to the sample's surface increases with the apparent contact angle; the maximum value is $1.783 \mathrm{~mm}$ at an apparent contact angle of $151.47^{\circ} \pm 1^{\circ}$.

As demonstrated in Fig. 9, the displacement of the droplet rapidly increases over the same time interval of $0.0625 \mathrm{~s}$, except for the etching time of $1 \mathrm{~min}$. The moving displacements of the droplets on the surface of the sample are $0,2.66,8.97,10.83$, 11.05 , and $11.69 \mathrm{~cm}$ at a time interval of $0.3125 \mathrm{~s}$. This corresponds to an etching time of 1, 5, 10, 15, 20 , and $25 \mathrm{~min}$, respectively. The moving displacement of the simulation is $2.02 \mathrm{~cm}$ at a time interval of
$0.125 \mathrm{~s}$. The moving displacement of the droplet is $1.85 \mathrm{~cm}$ at an etching time of $25 \mathrm{~min}$. These results show that the simulation is correct. As presented in Fig. 9, the angular velocity increases with an increased etching time at the same displacement. The angular velocities are 85.22, 287.66, 345.67, 353.87 , and $374.67 \mathrm{rad} / \mathrm{s}$ at a time interval of $0.3125 \mathrm{~s}$, which corresponds to etching time of $5,10,15,20$, and $25 \mathrm{~min}$, respectively. The resistance torque is decreased with an increase in the etching time; the minimum value is $0.091 \mathrm{mN} \cdot \mathrm{mm}$ for an etching time of $25 \mathrm{~min}$. In contrast, the angular acceleration increases with an increasing etching time; the maximum value is $1203.19 \mathrm{rad} / \mathrm{s}^{2}$, as demonstrated in Fig. 9. Because a droplet rolling on the super-hydrophobic surface must overcome the torques generated by the receding and advancing contact angles on the sample surfaces, if the gravitational torque is smaller than the sum of the torques generated by the hysteresis angles, the droplet will stay on the surface of the sample, as illustrated in Fig. 9(a). In contrast, according to Eq. (20), as presented in Fig. 9, the angular acceleration will increase with a decrease in the sum of the torques generated by the hysteresis angles. Therefore, the angular velocity of the droplet will increase faster on the super-hydrophobic surface. According to Eqs. (21)-(24), and as shown in Fig. 10(a), the calculated value is consistent with the measured value.

$$
m g \times \sin \varphi \times O H^{\prime} \geq 2\left(F_{\mathrm{RCA}}+F_{\mathrm{ACA}}\right) \int_{0}^{\pi / 2} r^{2} \sin \theta \mathrm{d} \theta
$$




$$
\begin{gathered}
\tau=I \times a \\
I=\int_{h}^{R} \frac{\pi \rho\left(R^{2}-h^{2}\right)}{2} \mathrm{~d} h \\
a=R \omega^{2} \\
\tau=m g \times \sin \varphi \times O H^{\prime}-2\left(F_{\mathrm{RCA}}+F_{\mathrm{ACA}}\right) \int_{0}^{\pi / 2} r^{2} \sin \theta \mathrm{d} \theta \\
\sin \varphi=k \frac{2 r \times \pi}{m g} \\
\pi^{2} \\
\operatorname{mg} \sin \varphi-F_{\mathrm{s}}=m a_{\mathrm{s}}
\end{gathered}
$$

where $a, I, \omega, g, a_{\mathrm{s}}, \varphi, \tau, F_{\mathrm{RCA}}$, and $F_{\mathrm{ACA}}$ are the angular acceleration, moment of inertia, angular velocity, gravity acceleration, sliding acceleration, inclination angle, torque, the adhesive force per unit of length corresponding to the RCA, and the adhesive force per unit of length corresponding to the ACA, respectively.

If the droplets are sliding on the super-hydrophobic surfaces. By considering Figs. 9, 10, and Eq. (27), the sliding resistance force $\left(F_{\mathrm{s}}\right)$ can be calculated. As shown in Fig. 11(a), the resistance force decreases with an increasing etching time; the minimum value is $0.004 \mathrm{mN}$ for an etching time of $25 \mathrm{~min}$. The resistance force is much lower compared to the sum of the values of the adhesive force per unit length of the RCA and ACA, as shown in Fig. 6(a) and in accordance with Eq. (24). Therefore, the $k$ is calculated by Eq. (26) when the droplet begins to slide on the surface sample, as shown in Fig. 11(a). Then, $F_{s}$ can be calculated according to $k$, as presented in Fig. 11(a). We determined that $F_{\mathrm{s}}$ is larger than the experimental resistance force. This is because when the droplet slides on the sample's surface, the droplet's translation motion occurs relative to the sample. Therefore, the contact area will participate in friction resistance, however, the droplet will roll on the surface of the sample. Thus, the resistance force will only be produced at the contact line of the droplet with the sample surface. This greatly reduces the drag of the droplet motion. Therefore, the droplets will roll due to very small external forces. Hence, the droplets rolling on the surface must occur at a higher contact angle surface, however, the surface with higher contact angles is not sufficient for droplet rolling because it is not only related to the contact angle, but also the chemical composition of the sample surface. The droplet will roll on the surface when the gravitational torque is greater than the sum of the torques generated by the hysteresis angles. Conversely, the droplet may remain on the sample's surface or it may slide.
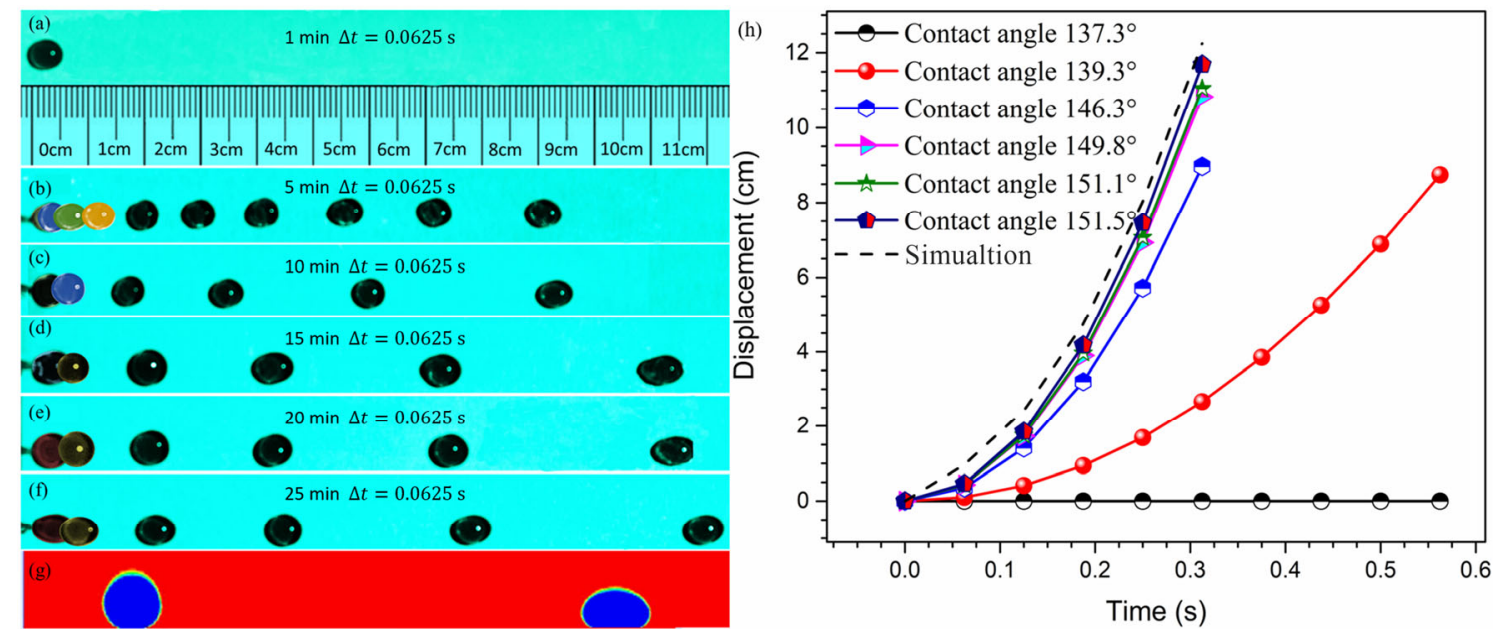

Fig. 9 Different displacements of a droplet on the surface of the sample at different time measured by a high-speed camera: (a) $1 \mathrm{~min}$, (b) $5 \mathrm{~min}$, (c) $10 \mathrm{~min}$, (d) $15 \mathrm{~min}$, (e) $20 \mathrm{~min}$, and (f) $25 \mathrm{~min}$, respectively; (g) simulation and (h) displacement of a droplet as a function of time. 

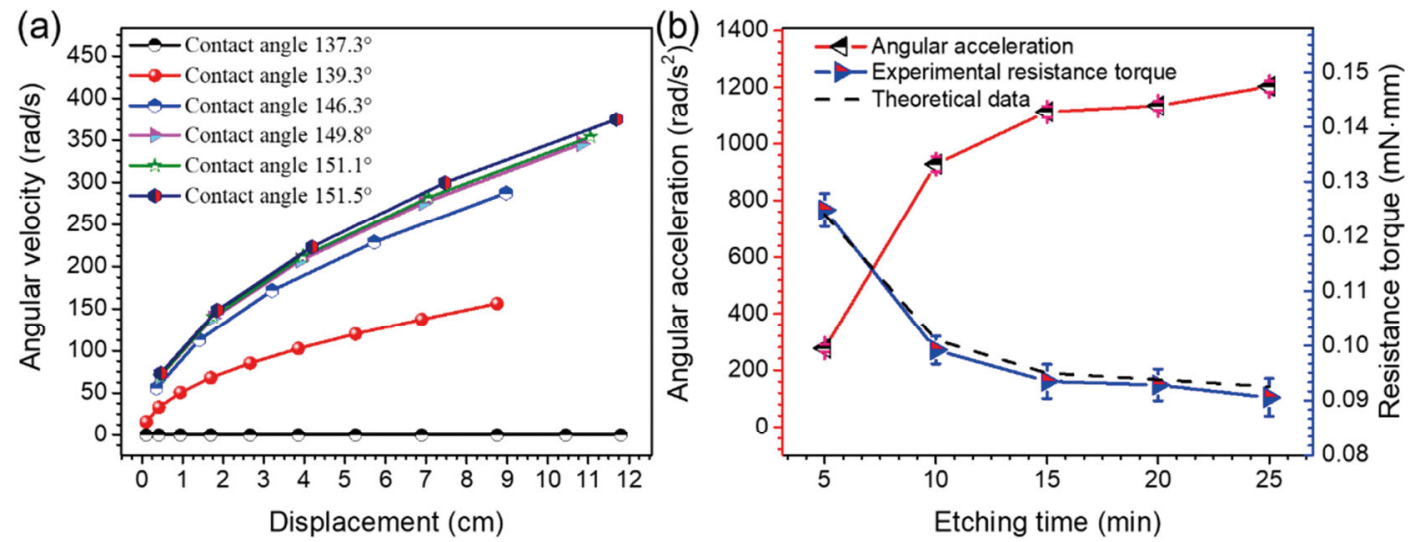

Fig. 10 (a) Angular velocity as a function of the displacement of a droplet on a sample. (b) The average angular acceleration is plotted on the left $y$-axis, and the resistance torque is plotted on the right $y$-axis as a function of the etching time.
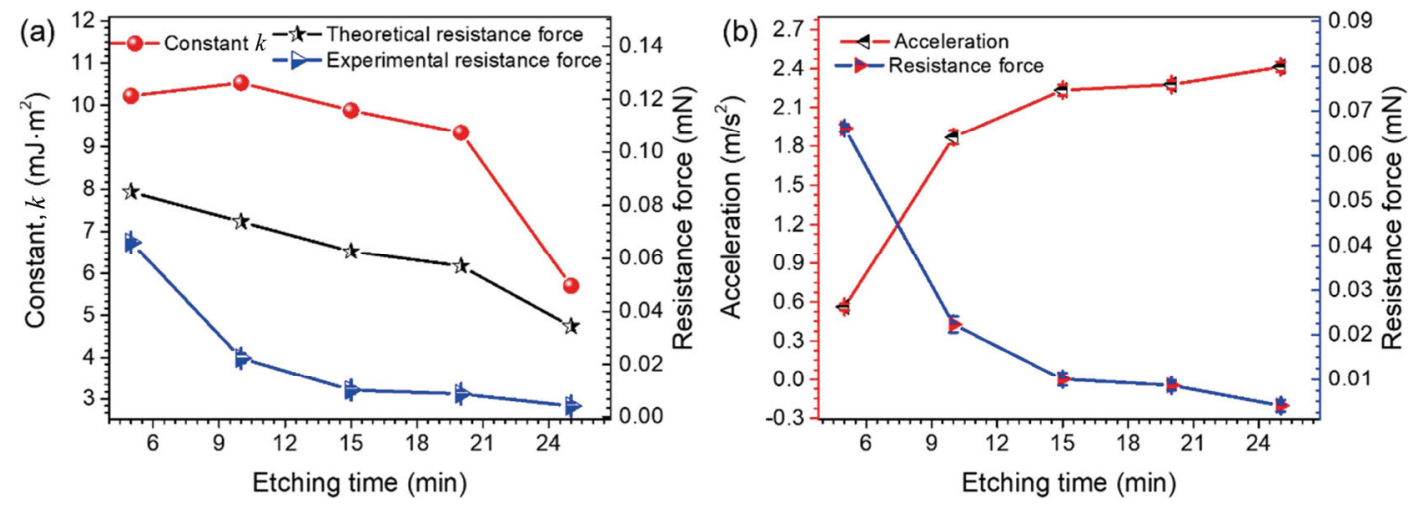

Fig. 11 (a) $k$ is plotted on the left $y$-axis and the experimental and theoretical [43] sliding resistance force is plotted on the right $y$-axis as a function of the etching time. (b) The acceleration is plotted on the left $y$-axis and the sliding resistance force is plotted on the right $y$-axis as a function of the etching time.

\section{Conclusions}

In order to study the droplet motion on superhydrophobic surfaces, super-hydrophobic surfaces with a varied surface roughness were fabricated and the resistance force for rolling on the rough surfaces was evaluated. In the highly hydrophobic region, the resistance force for rolling decreased with an increasing contact angle, which is considered to be dependent on the surface roughness. Microstructural observations revealed that the surface structure can trap air, which is important for decreasing the rolling resistance force.

For the droplet's motion on the surface, an equation is proposed to describe the relationship between the rolling resistance force and the contact angles on the super-hydrophobic surfaces with a varied surface roughness. The results calculated based on this equation agree well with the experimental results.
At the same time, the adhesive force that corresponds to the ACA and the RCA is measured with a dynamic contact angle measuring device and a tensiometer with a high-speed camera. This study proved that the droplet on the super-hydrophobic surface has a rolling motion. This study is only applicable to a droplet super-hydrophobic surface with low adhesion. However, the relationship between the entrapped air stability and the adhesive force of the ACA and the RCA at different velocities, still need a lot of studies, when the super-hydrophobic surface placed in the flow field.

\section{Acknowledgements}

The authors acknowledge the financial support from the National Natural Science Foundation of China (Grant Nos. U1809214 and U1809213), the 
National Basic Research Program of China (973 Program) (Grant No. 2013CB632302), the Foundation of Key Laboratory of Marine Materials and Related Technologies, Chinese Academy of Sciences (2018K01), and the Foundation of Key Laboratory of Marine Materials and Related Technologies, CAS (2018K01).

Open Access This article is licensed under a Creative Commons Attribution 4.0 International License, which permits use, sharing, adaptation, distribution and reproduction in any medium or format, as long as you give appropriate credit to the original author(s) and the source, provide a link to the Creative Commons licence, and indicate if changes were made.

The images or other third party material in this article are included in the article's Creative Commons licence, unless indicated otherwise in a credit line to the material. If material is not included in the article's Creative Commons licence and your intended use is not permitted by statutory regulation or exceeds the permitted use, you will need to obtain permission directly from the copyright holder.

To view a copy of this licence, visit http://creativecommons.org/licenses/by/4.0/.

\section{References}

[1] Li L Y, Zhu J F, Zhi S D, Liu E Y, Wang G, Zeng Z X, Zhao W J, Xue Q J. Study of adhesion and friction drag on a rough hydrophobic surface: Sandblasted aluminum. Phys Fluids 30(7): 071903 (2018)

[2] Fuaad P A, Arul Prakash K. Enhanced drag-reduction over superhydrophobic surfaces with sinusoidal textures: A DNS study. Comput Fluids 181: 208-223 (2019)

[3] Lu Y. Superior lubrication properties of biomimetic surfaces with hierarchical structure. Tribol Int 119: 131-142 (2018)

[4] Xiang T F, Han Y, Guo Z Q, Wang R, Zheng S L, Li S, Li C, Dai X M. Fabrication of inherent anticorrosion superhydrophobic surfaces on metals. ACS Sustainable Chem Eng 6(4): 5598-5606 (2018)

[5] Yang Z Q, Wang L D, Sun W, Li S J, Zhu T Z, Liu W, Liu G C. Superhydrophobic epoxy coating modified by fluorographene used for anti-corrosion and self-cleaning. Appl Surf Sci 401: 146-155 (2017)

[6] Wang Z W, Su Y L, Li Q, Liu Y, She Z X, Chen F N, Li L Q, Zhang X X, Zhang P. Researching a highly anti- corrosion superhydrophobic film fabricated on AZ91D magnesium alloy and its anti-bacteria adhesion effect. Mater Charact 99: 200-209 (2015)

[7] Zeng W G, Chen J, Yang H, Deng L D, Liao G F, Xu Z S Robust coating with superhydrophobic and self-cleaning properties in either air or oil based on natural zeolite. Surf Coat Technol 309: 1045-1051 (2017)

[8] Latthe S S, Sutar R S, Kodag V S, Bhosale A K, Kumar A M, Kumar S K, Xing R M, Liu S H. Self-cleaning superhydrophobic coatings: Potential industrial applications. Prog Org Coat 128: 52-58 (2019)

[9] Lei S, Wang F J, Fang X Z, Ou J F, Li W. Icing behavior of water droplets impinging on cold superhydrophobic surface. Surf Coat Technol 363: 362-368 (2019)

[10] Fang Y, Yong J L, Chen F, Huo J L, Yang Q, Zhang J Z, Hou X. Bioinspired fabrication of $\mathrm{Bi} /$ tridirectionally anisotropic sliding superhydrophobic PDMS surfaces by femtosecond laser. Adv Mater Interfaces 5(6): 1701245 (2018)

[11] Richard D, Quéré D. Viscous drops rolling on a tilted non-wettable solid. EPL 48(3): 286-291 (1999)

[12] Raimondo M, Veronesi F, Boveri G, Guarini G, Motta A, Zanoni R. Superhydrophobic properties induced by sol-gel routes on copper surfaces. Appl Surf Sci 422: 1022-1029 (2017)

[13] Yang M P, Liu W Q, Jiang C, He S, Xie Y K, Wang Z F. Fabrication of superhydrophobic cotton fabric with fluorinated $\mathrm{TiO}_{2}$ sol by a green and one-step sol-gel process. Carbohydr Polym 197: 75-82 (2018)

[14] Xie A T, Cui J Y, Chen Y Y, Lang J H, Li C X, Yan Y S, Dai J D. One-step facile fabrication of sustainable cellulose membrane with superhydrophobicity via a solgel strategy for efficient oil/water separation. Surf Coat Technol 361: 19-26 (2019)

[15] Kim J H, Mirzaei A, Kim H W, Kim S S. Facile fabrication of superhydrophobic surfaces from austenitic stainless steel (AISI 304) by chemical etching. Appl Surf Sci 439: 598-604 (2018)

[16] Zhang X, Zhao J, Mo J L, Sun R Y, Li Z, Guo Z G. Fabrication of superhydrophobic aluminum surface by droplet etching and chemical modification. Colloids Surf A: Physicochem Eng Aspects 567: 205-212 (2019)

[17] Kumar A, Gogoi B. Development of durable self-cleaning superhydrophobic coatings for aluminium surfaces via chemical etching method. Tribol Int 122: 114-118 (2018)

[18] Zheng X M, Fu S Y. Reconstructing micro/nano hierarchical structures particle with nanocellulose for superhydrophobic coatings. Colloids Surf A: Physicochem Eng Aspects 560: 171-179 (2019)

[19] Li S Y, Xiang X G, Ma B H, Meng X D. Facile preparation of diverse alumina surface structures by anodization and superhydrophobic surfaces with tunable 
water droplet adhesion. J Alloys Compd 779: 219-228 (2019)

[20] Zhou X, Yu S R, Guan S W, Lv Z X, Liu E Y, Zhao Y. Fabrication and characterization of superhydrophobic $\mathrm{TiO}_{2}$ nanotube coating by a facile anodic oxidation approach. Surf Coat Technol 354: 83-91 (2018)

[21] Duron C M, Zhong J, David A E, Ashurst W R, Bhavnani S H, Morris J R, Bates A C. Development of a durable vapor phase deposited superhydrophobic coating for steam cycle power generation condenser tubes. Journal of Thermal Science and Engineering Applications 10(5): 054501 (2018)

[22] Tan R X, Xie H Y, She J Q, Liang J S, He H, Li J H, Fan Z Q, Liu B. A new approach to fabricate superhydrophobic and antibacterial low density isotropic pyrocarbon by using catalyst free chemical vapor deposition. Carbon 145: 359-366 (2019)

[23] Yuan Z P, Hu Z F, Chu F Q, Wu X M. Enhanced and guided self-propelled jumping on the superhydrophobic surfaces with macrotexture. Appl Phys Lett 115(16): 163701 (2019)

[24] Yuan Z P, Hu Z F, Gao S H, Wu X M. The effect of the initial state of the droplet group on the energy conversion efficiency of self-propelled jumping. Langmuir 35(48): 16037-16042 (2019)

[25] Li J, Du F, Zhao Y H, Zhao S C, Yu H D. Two-step fabrication of superhydrophobic surfaces with anti-adhesion. Opt Laser Technol 113: 273-280 (2019)

[26] Wang Y Q, Shi H X, Li X G. Revisiting the fabrication of superhydrophobic aluminum surfaces and their use as soft substrates for droplet manipulation. J Mater Sci 54(10): 7469-7482 (2019)

[27] Marmur A, Kojevnikova S. Super-hydrophobic surfaces: Methodological considerations for physical design. $J$ Colloid Interface Sci 568: 148-154 (2020)

[28] Shi X T, Zhu Y L, Liu Y H, Feng L B. Comparative study of structure and property changes in corrosive media for self-cleaning superhydrophobic magnesium alloys. $J$ Wuhan Univ Technol-Mater Sci Ed 34(2): 410-416 (2019)

[29] Yao C W, Tang S R, Sebastian D, Tadmor R. Sliding of water droplets on micropillar-structured superhydrophobic surfaces. Appl Surf Sci 504: 144493 (2020)

[30] Zhou X K, Xue W, Liu W W, Zhu D H, Cao Y. Quadri-directionally anisotropic droplets sliding surfaces fabricated by selective laser texturing of aluminum alloy plates. Appl Surf Sci 509: 145406 (2020)
[31] Liang L S, Lu L S, Xing D, Wan Z P, Tang Y. Preparation of superhydrophobic and anti-resin-adhesive surfaces with micro/nanoscale structures on high-speed steel via laser processing. Surf Coat Technol 357: 57-68 (2019)

[32] Ning N Y, Wang S, Zhang Z T, Feng Z B, Zheng Z P, Yu B, Tian M, Zhang Liqun. Superhydrophobic coating with ultrahigh adhesive force and good anti-scratching on elastomeric substrate by thiol-ene click chemistry. Chem Eng J 373: 318-324 (2019)

[33] Hao P F, Lv C J, Yao Z H, He F. Sliding behavior of water droplet on superhydrophobic surface. EPL 90(6): 66003 (2010)

[34] Su X J, Li H Q, Lai X J, Zhang L, Liao X F, Wang J, Chen $\mathrm{Z} \mathrm{H}, \mathrm{He} J$, Zeng $\mathrm{X}$ R. Dual-functional superhydrophobic textiles with asymmetric roll-down/ pinned states for water droplet transportation and oil-water separation. ACS Appl Mater Interfaces 10(4): 4213-4221 (2018)

[35] Ran M H, Yang C X, Fang Y, Zhao K K, Ruan Y Q, Wu J, Yang H, Liu Y F. Model for rolling angle. J Phys Chem C 116(15): 8449-8455 (2012)

[36] Keiser A, Keiser L, Clanet C, Quéré D. Drop friction on liquid-infused materials. Soft Matter 13(39): 6981-6987 (2017)

[37] Miwa M, Nakajima A, Fujishima A, Hashimoto K, Watanabe T. Effects of the surface roughness on sliding angles of water droplets on superhydrophobic surfaces. Langmuir 16(13): 5754-5760 (2000)

[38] Feng L, Li S, Li Y, Li H, Zhang L, Zhai J, Song Y, Liu B, Jiang L, Zhu D. Super-hydrophobic surfaces: From natural to artificial. Adv Mater 14(24): 1857-1860 (2002)

[39] Wenzel R N. Resistance of solid surfaces to wetting by water. Ind Eng Chem 28(8): 988-994 (1936)

[40] Cassie A B D, Baxter S. Wettability of porous surfaces. Trans Faraday Soc 40: 546-551 (1944)

[41] Tadmor R. Line energy and the relation between advancing, receding, and young contact angles. Langmuir 20(18): 7659-7664 (2004)

[42] Chibowski E, Terpilowski K. Surface free energy of sulfur-revisited: I. Yellow and orange samples solidified against glass surface. J Colloid Interface Sci 319(2): 505-513 (2008)

[43] Furmidge C G L. Studies at phase interfaces. I. The sliding of liquid drops on solid surfaces and a theory for spray retention. J Colloid Sci 17(4): 309-324 (1962) 


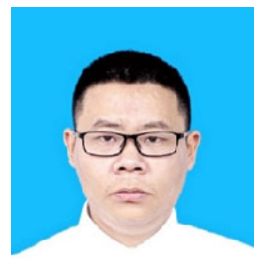

Longyang LI. He received his Ph.D. degree in materials processing engineering from the Ningbo Institute of Materials Technology and Engineering, Chinese Academy of Sciences (CAS), in 2019. Then, he joined the Key Laboratory of

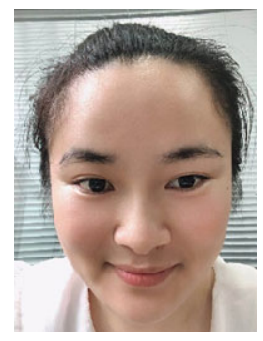

Jingfang ZHU. She received her master degree in materials processing engineering in 2017 from the North University of China, Taiyuan, China. She is now a Ph.D. student in the

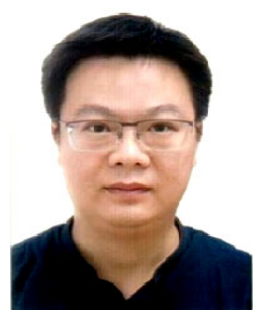

Zhixiang ZENG. He received his $\mathrm{Ph} . \mathrm{D}$. degree in materials science from the Lanzhou Institute of Chemical Physics, CAS, in 2009. Then, he joined the Key Laboratory of Marine Materials and Related Technologies at Ningbo Institute

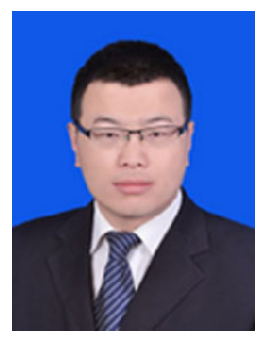

Eryong LIU. He received his Ph.D. degree in materials science and engineering from $\mathrm{Xi}^{\prime}$ an Jiaotong University, 2013. Then, he joined the School of Materials Science

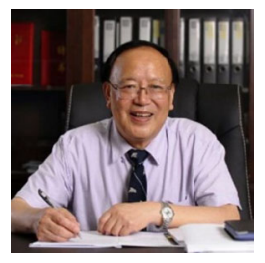

Qunji XUE. He received his master degree in materials science from the Lanzhou Institute of Chemical Physics, CAS, in 1967. Since 2010, he joined the Key Laboratory of Marine Materials and Related Technologies at Ningbo Institute of Materials Technology and Engineering, CAS. His current position is an
Marine Materials and Related Technologies at Ningbo Institute of Materials Technology and Engineering, CAS. His current position is as a research assistant in the laboratory. His research areas cover the tribology of composite materials and tribology of bionic.

Key Laboratory of Micro-Electro-Mechanical System (MEMS) of Ministry of Education, Southeast University, Nanjing, China. Her research interests focus on oil-water separation.

of Materials Technology and Engineering, CAS. His current position is as a professor. His research areas cover interface control and bionic drag reduction of the marine vehicle, electrochemical chemical deposition and corrosion and wear characteristics of the coating, and marine oil pollution treatment and oil-water separation materials.

and Engineering, Xi'an University of Science and Technology. His current position is as an associate professor. His research areas cover the surface engineering and materials performance.

academician of the Chinese Academy of Engineering. He created the Key Lab of Marine Materials and Application technologies. And now, besides tribology, marine materials and technologies such as drag reduction and noise reduction technology, surface enhancement of key parts for marine equipments, oil absorption materials, antifouling paints, and anticorrosive paints, are also his research interests. 